\title{
Comparison of some Algorithms in Image Compression
} Application Manar y. Ahmed

College of Computer Science and Mathematics University of Mosul, Iraq

Received on: $16 / 12 / 2002$

Accepted on: 30/06/2003

\section{ABSTRACT}

Today there are a number of algorithms developed in the framework of international committees that allow still image compression. In this paper, the area of Vector Quantization (VQ) neural network with the Self-Organizing Feature Map (SOFM) has been compared with the ordinary vector Quantization technique Linde-Buzo-Gray (LBG) in image compression. The results were compared with the Back Propagation Neural Network BPNN which was employed to design a code book of an image to be compressed using VQ method. Results show that the neural technique gives a performance that is very close to optimal. The BPNN scheme not only has the advantage of the SOFM - VQ scheme but also improves the coded image quality. Experimental results are given and comparisons are made using the BPNN coding scheme and some other coding techniques. In the experiments, the BPNN coding scheme achieves the better visual quality about edge region and the best peak signal-to-noise ratio PSNR performance at nearly the same bit rate.

Keywords: vector Quantization technique (VQ), Linde-BuzoGray (LBG), image compression, Back Propagation Neural Network BPNN. 


$$
\begin{aligned}
& \text { تطبيق مقارنة بعض خوارزميات كبس الصور } \\
& \text { منار يونس احمد } \\
& \text { كلية علوم الحاسبات والرياضيات } \\
& \text { جامعة الدوصل } \\
& \text { تاريخ استلام البحث: 2002/12/16 ت اريخ قبول البحث: 2003/06/30 } \\
& \text { الملخص }
\end{aligned}
$$

هناك اليوم عدد من الخوارزميات التي تطورت في هيكل اللجان الدولية التي

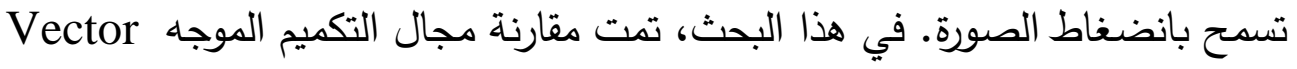

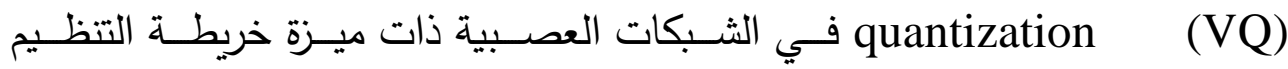
(SOFM) تقنية التكميم الموجه العادي (LBG) Linde -Buzo- Gray) في كبس الصور • وقورنت النتائج أيضـا مـع الشبكة العصبية ذات الانتشار الخلفي BPNN- Back Propagation Neural Network كتاب الثفرة من الصورة المراد ضغطها باستخدام طريقة التكميم الموجه. تبين النتائج أن التقنيات العصبية تمنح الأداء الذي يكون قريبا من المثالي. لا تمتلك الثبكة العصبية BPNN فقط منافع SOFM-VQ ولكن أيضـا تحسن

نوعية الصورة المشفرة. أعطيت نتائج التجارب وقدمت المقارنات بين BPNN وبعض تقنيات التثفير الأخرى. في التجارب، المشفر

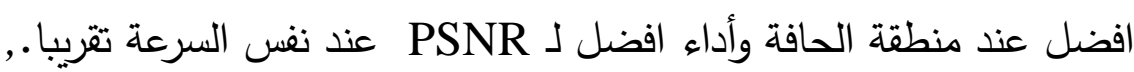



\section{Introduction}

$$
\text { الصورة، الثبكة العصبية ذات الانتثار الخلفي BPNN. }
$$

The transport of images across communication paths is an expensive process. Image compression provides an option for reducing the number of bits in transmission. This in turn helps increase the volume of data transferred in a space of time, along 
with reducing the cost required. It has become increasingly important to most computer networks as the volume of data traffic has begun to exceed their capacity for transmission. Artificial Neural networks have found increasing applications in this field due to their noise suppression and learning capabilities. There are different algorithms for image compression, one of these algorithms have been developed on the basis of a modular structured neural network which consists of multiple neural network with different block size (the number of input units) for the region segmentation [12]. Multilayer neural networks have been developed [1] as another image compression algorithm, in which the use of two-layer neural network had been extended to Multilayer network. Also Auto associative transform coding approach which employs two-layer feed forward neural network to compress images has been compared to the new technique of four or more layers which in turn needs along time to be trained [2]. A self organizing neural network has been used in which vector quantization learning rule has employed the possibility of applying self-organizing maps to the problem of vector quantization is discussed by Kohonen (1990). The selforganizing neural network architecture is based on a twodimensional lattice formed by interconnected processing elements. Such a lattice receives its input through a relay layer. It is a simple fan-out of the input samples to each of the processing elements of the lattice [5].

In this work, LBG algorithm and feed forward back propagation algorithm have been employed to achieve the code book for VQ. Vector quantization based on neural processing with SOFM has also been applied to image. Finally a comparison between them has been made.

\section{Image compression with Vector quantization}

Vector quantization (VO) has been found to be an efficient method for still image coding. The image to be encoded is first partitioned into non-overlapping blocks to yield a set of vectors. 
The input vectors are individually quantized to the closest code word in the code book, which is generated by using some clustering algorithms such as the Linde-Buzo-Gray (LBG) algorithm. The LBG algorithm is an iterative method based on the Lloyd clustering algorithm. Given an initial partitioning of the set of training vectors, the goal of the algorithm is to classify them into clusters and to compute a centroid, that is, the representative of the elements of the cluster. The classification process is performed on the bases of a metric defined on the set of training vector and the nearest-neighbor rule. The centroids are it eratively updated whenever a new element is added to the cluster that they represent. Each iteration goes through all the elements of the training set. The stopping criterion is based on a predefined distortion measure. If the average distortion at the current iteration is under a predefined threshold, the classification process is stopped. For image applications, the Mean Sequare Error (MSE) is used as the distortion measure.

Where

$$
\operatorname{MSE}=(1 / n)^{2} \sum_{\mathrm{i}=1}^{\mathrm{n}} \sum_{\mathrm{j}=1}^{\mathrm{n}}\left(\mathrm{xij}-^{\wedge} \mathrm{xij}\right)^{2}
$$

Where $\mathrm{xij}$ and ${ }^{\wedge} \mathrm{xij}$ denote the original and quantized gray levels, respectively. Image compression is achieved by using the indices of code words for the information of the encoded images. Image decompression is done by utilizing the indices as addresses to the corresponding code words in the decoder's code book. This process can be implemented by table lookup techniques [6].

\section{Artificial Neural Network for Image compression}

Artificial Neural Networks (ANNs) have been applied to many problems [3], and have demonstrated their superiority over traditional methods when dealing with noisy or incomplete data. One such application is for image compression. Neural networks seem to be well suited to this particular function, as they have the 
ability to preprocess input patterns to produce simpler patterns with fewer components [4]. This compressed information (stored in a hidden layer) preserves the full information obtained from the external environment. Not only can ANN based techniques provide sufficient compression rates of the data in question, but security is easily maintained. This occurs because the compressed data that is sent along a communication line is encoded and not resemble its original form. There have already been an exhaustive number of papers published applying ANNs to image compression [7]. Many different training algorithms and architectures have been used. Some of the more notable in the literature are: nested training algorithms used with symmetrical multilayer neural network, self organizing maps, for code book generation [9], principal component analysis network, back propagation network [11], and the adaptive principal component extraction algorithm [10].

\section{BPNN for VQ Code Book design}

In this paper, a BPNN for VQ Code Book design has been employed. The technique was based on using the BPNN to train the three layered neural network for designing the code book of an image to be compressed using VQ method.

\section{Self-Organization Feature Map for VQ (SOFM - VQ)}

SOFM network for VQ classifies input vector into classes by using a competitive layer to find subclasses of input vectors, and then combining them into the target classes. Unlike perceptrons, this network can classify any set of input vectors, not just linearly separable sets of input vectors. The only requirement is that the competitive layer must have enough neurons, and each class must be assigned enough competitive neurons [8]. 


\section{Simulations and Results}

In the simulations, a two- layer BPNN was used. For the back- propagation learning algorithm the initial synaptic weights are produced by a random number generation function at a floating interval [0-0.00001].The learning rate parameter $\eta=$ 0.03 is chosen to produce the final synaptic weight vectors because of the tradeoff between the prediction performance and computational speed. The master code book is generated by the LBG algorithm from the training set of five different image. The test images are monochrome images of size 512x 512 with 256 gray levels. To evaluate the coder's performance numerically, the peak signal-to-noise ratio(PSNR) between the image and the encoded image has been calculated, where the PSNR is defined as

$$
\mathrm{PSNR}=10 \log ^{10}(255)^{2} / \mathrm{MSE} \mathrm{dB}
$$

Three coding schemes has been compared, the ordinary VQ the SOFM-VQ, and the BPNN in the simulation. In the ordinary $\mathrm{VQ}$, the size of the input blocks is $4 \times 4$ and the code book size is 32. In the SOFM-VQ scheme, the master code book size is 512 , the state code book size is 15 , and the dimension of the input vectors is 16 . The ordinary VQ is a fixed bit-rate coding scheme. Hence, a number of thresholds has been tried for each image in the simulations in order to compare the PSNR values of the images that are encoded by these three coding schemes at nearly the same bit rate. Note that the threshold can be adjusted to obtain the desired image quality and bit rate. Table 1 shows the PSNR values, the bit rates, and the number of vectors with full searching of simulation results for the images in the training set. In the experiments, the improvements over the ordinary VQ is up to $3.62 \mathrm{~dB}$ and the improvement over the SOFM-VQ is up to $1.59 \mathrm{~dB}$ for the image Lena. 
TABLE 1

The PSNR Values, Bit Rates, and Number of Vectors with Full Searching (Nf) of the Ordinary VQ, SOFM- VQ, and BPNN Schemes for the images in the Training Set .

\begin{tabular}{|c|c|c|c|c|c|c|c|c|}
\hline & \multicolumn{2}{|c|}{ Ordinary VQ } & \multicolumn{3}{c|}{ SOFMVQ } & \multicolumn{3}{c|}{ BPNN } \\
\hline Images & PSNR & Bit rate & PSNR & Bit rate & N $f$ & PSNR & Bit rate & N $f$ \\
\hline Peppers & $\mathbf{2 8 . 3 7}$ & $\mathbf{0 . 3 1 2 5}$ & $\mathbf{3 0 . 4 0}$ & $\mathbf{0 . 3 0 8 8}$ & $\mathbf{1 7 1 2}$ & $\mathbf{3 1 . 9 9}$ & $\mathbf{0 . 3 0 9 1}$ & $\mathbf{1 7 2 2}$ \\
\hline Airplane & $\mathbf{2 8 . 0 4}$ & $\mathbf{0 . 3 1 2 5}$ & $\mathbf{2 9 . 1 9}$ & $\mathbf{0 . 3 0 5 4}$ & $\mathbf{1 6 1 5}$ & $\mathbf{3 0 . 7 5}$ & $\mathbf{0 . 3 0 5 3}$ & $\mathbf{1 6 1 2}$ \\
\hline Sailboat & $\mathbf{2 6 . 5 9}$ & $\mathbf{0 . 3 1 2 5}$ & $\mathbf{2 7 . 0 2}$ & $\mathbf{0 . 3 1 1 9}$ & $\mathbf{1 8 0 4}$ & $\mathbf{2 8 . 7 0}$ & $\mathbf{0 . 3 1 2 5}$ & $\mathbf{1 8 2 0}$ \\
\hline Boat & $\mathbf{2 7 . 0 5}$ & $\mathbf{0 . 3 1 2 5}$ & $\mathbf{2 8 . 1 5}$ & $\mathbf{0 . 3 1 1 6}$ & $\mathbf{1 7 9 4}$ & $\mathbf{2 9 . 3 5}$ & $\mathbf{0 . 3 1 1 7}$ & $\mathbf{1 7 9 6}$ \\
\hline Tiffany & $\mathbf{2 8 . 8 6}$ & $\mathbf{0 . 3 1 2 5}$ & $\mathbf{3 2 . 1 4}$ & $\mathbf{0 . 2 9 3 1}$ & $\mathbf{1 2 5 4}$ & $\mathbf{3 3 . 3 8}$ & $\mathbf{0 . 2 9 4 5}$ & $\mathbf{1 2 9 6}$ \\
\hline
\end{tabular}

Table 2 shows the PSNR values, the bit rates, and the number of vectors with full searching of simulation results for the images outside the training set.

The improvement over the ordinary VQ is up to $2.94 \mathrm{~dB}$ and the improvement over the SOFM-VQ is up to $1.63 \mathrm{~dB}$ for the image Lena. Moreover, we also compare the number of full search vectors for the reconstructed image Lena of both SOFMVQ and BPNN at nearly the same PSNR value. By using the SOFM-VQ scheme, all 1743 full search vectors are used to encode the image Lena and PSNR value is $29.5 \mathrm{~dB}$. For the same quality encoded image, only 612 full search vectors are needed to encode the image by using the BPNN. That is, the BPNN can achieve a better accuracy of side- match prediction than the SOFM-VQ.

\section{TABLE 2}

The PSNR Values, and Number of Vectors with Full Searching (N $f$ ) of the Ordinary VQ, SOFM-VQ, and BPNN Scheme for Images outside the Training Set .

\begin{tabular}{|l|l|l|l|l|l|l|l|c|}
\hline & Ordinary VQ & \multicolumn{3}{c|}{ SOFMVQ } & \multicolumn{3}{c|}{ BPNN } \\
\hline Images & PSNR & Bit rate & PSNR & Bit rate & N $f$ & PSNR & Bit rate & N $f$ \\
\hline Lena & 28.22 & 0.3125 & 29.53 & 0.3098 & 1743 & 31.16 & 0.3086 & 1707 \\
\hline Family & 27.01 & 0.3125 & 27.68 & 0.3121 & 1809 & 28.94 & 0.3122 & 1813 \\
\hline Zelda & 30.25 & 0.3125 & 32.54 & 0.3064 & 1643 & 33.72 & 0.3057 & 1594 \\
\hline
\end{tabular}

The images Peppers and Lena in fig. 2a are the original 512x 512 monochrome images with 8 bit per pixel (bpp). Figures 
1b- 1d show the encoded results for the image Peppers, which is the training set. Figure $1 \mathrm{~b}$ shows the encoded image by using the ordinary VQ scheme at 0.3125 bpp. Figure 1c is the encoded result of the SOFM-VQ scheme at $0.3088 \mathrm{bpp}$. The BPNN yield the result shown in fig. 1d, which is encoded at $0.3091 \mathrm{bpp}$.

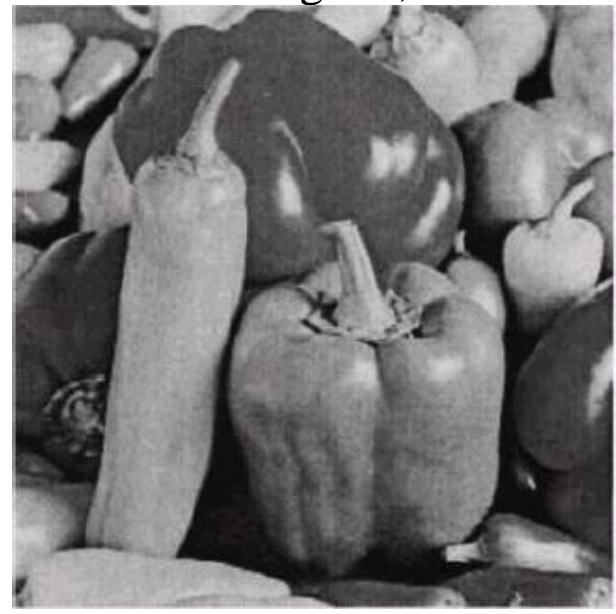

(a)

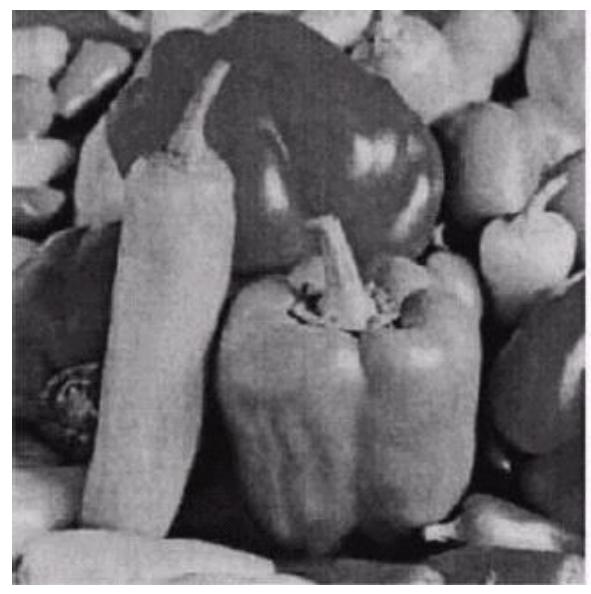

(c)

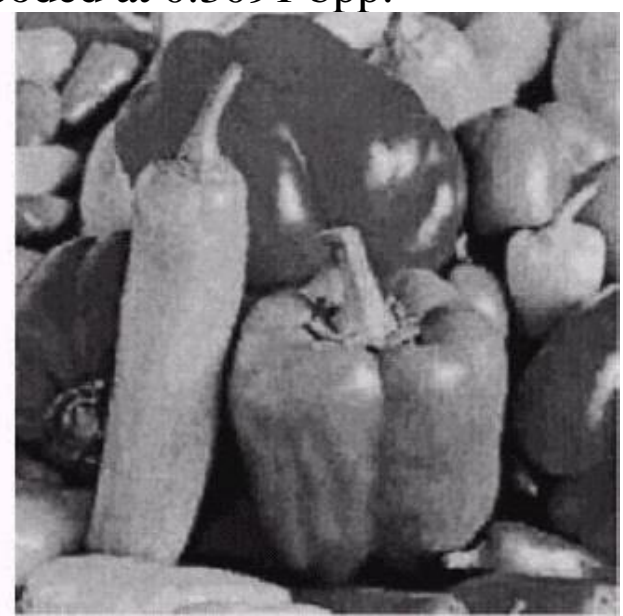

(b)

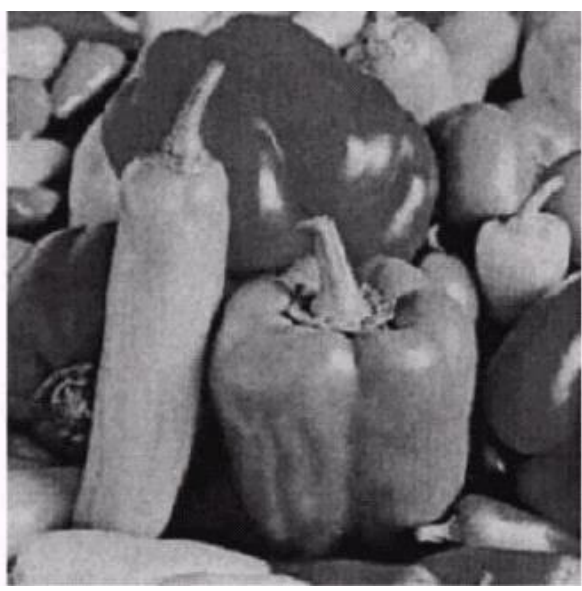

(d)

FIG.1. Results of the image Peppers: (a) original image, (b) VQ at $0.3125 \mathrm{bpp}$, (c) SOFM-VQ at $0.3088 \mathrm{bpp}$, and (d) BPNN scheme at 0.3091 bpp. 
Figures $2 b-2 d$ show the encoded results for the image Lena, which is outside the training set. Figure $2 b$ shows the encoded image by using an ordinary VQ scheme at $0.3125 \mathrm{bpp}$. Figure $2 c$ is the encoded result of the SOFM-VQ and the bit rate is $0.3098 \mathrm{bpp}$. Figure $2 \mathrm{~d}$ shows the encoded result of the BPNN for the image Lena and the average bit rate is $0.3086 \mathrm{bpp}$.

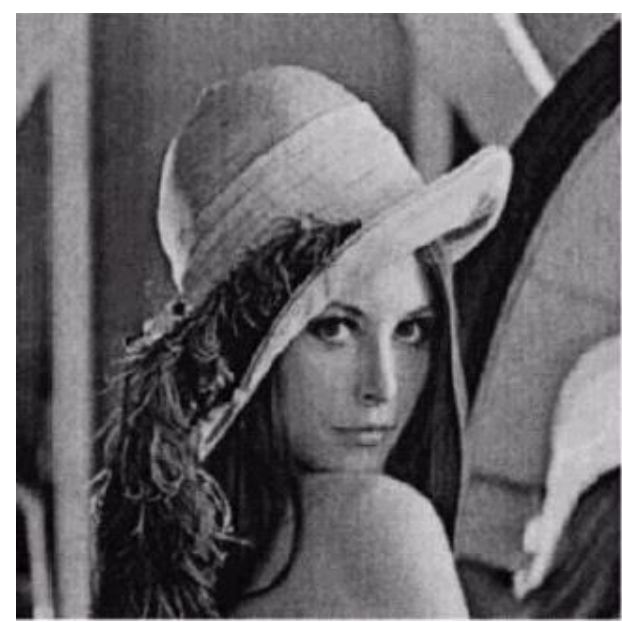

(a)

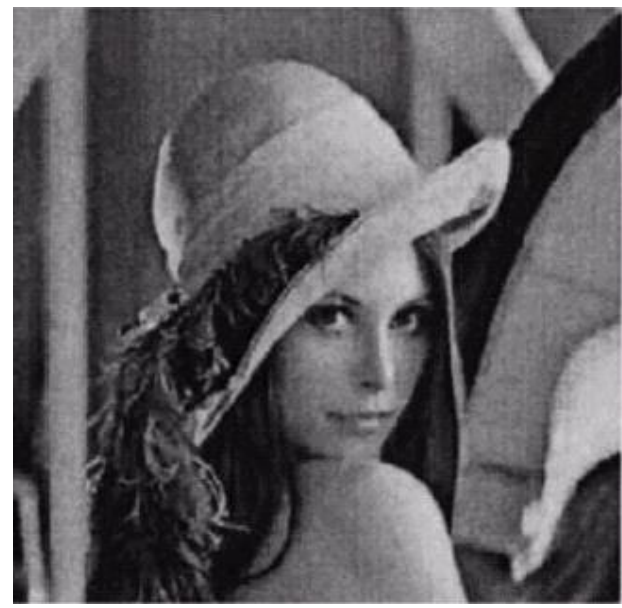

(c)

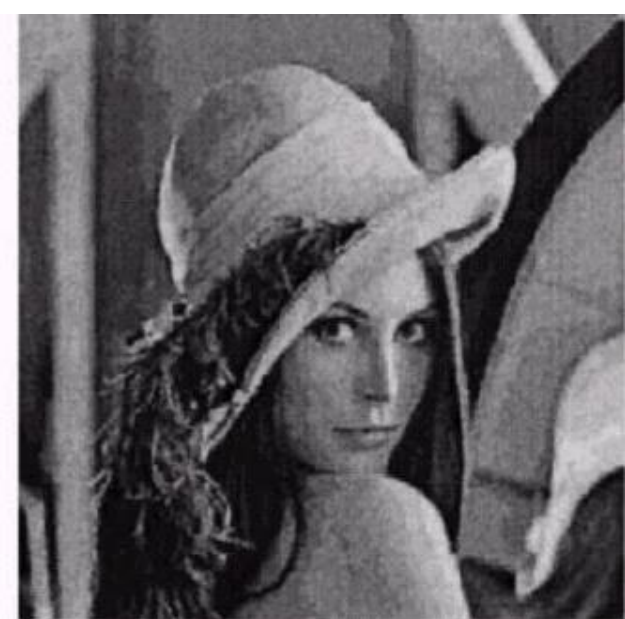

(b)



(d) 
FIG.2. Results of the image Lena : (a) original image, (b) VQ at $0.3125 \mathrm{bpp}$, (c) SOFM- VQ at $0.3098 \mathrm{bpp}$, and (d) BPNN scheme at $0.3086 \mathrm{bpp}$.

To show the differences clearly, the enlarged portion in the processed images Lena is given in fig. $3 a$ and fig $3 b$. It can be seen easily that our proposed scheme can obtain the better image quality and the better visual quality about a fine region. Table 3 shows the improvement of PSNR values over the ordinary VQ and SOFM-VQ of simulation results at nearly the same bit rate .

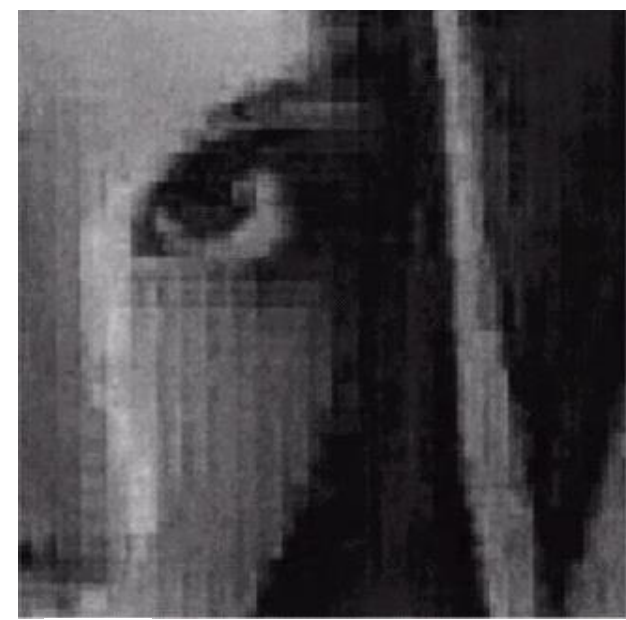

(a)

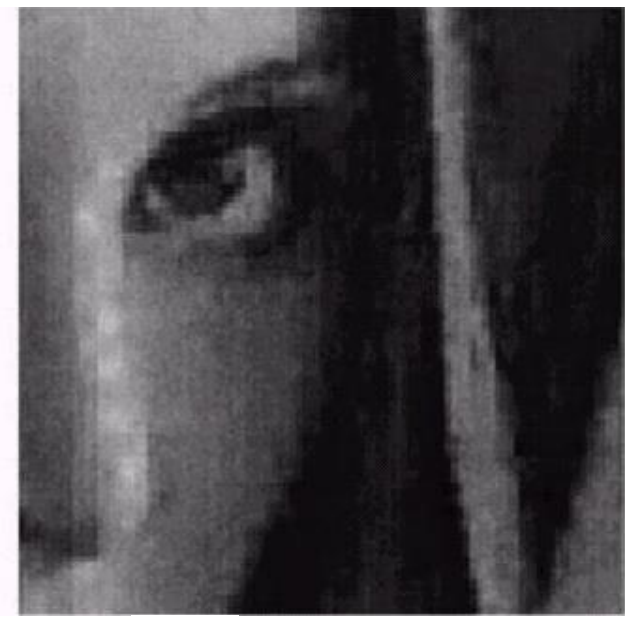

(b)

FIG.3. Results of the image Lena : (a) magnified portion of the reconstruction image fig. $2 c$ and (b) magnified portion of the reconstruction image fig. $2 \mathrm{~d}$.

TABLE 3

The PSNR Improvement over the Ordinary VQ and SOFMVQ Scheme at Nearly the Same Bit Rate 0.31 bpp

\begin{tabular}{|c|c|c|c|}
\hline & Images & Over VQ & Over SOFM-VQ \\
\hline Inside & Peppers & 3.62 & 1.59 \\
\hline & Airplane & 2.71 & 1.56 \\
\hline & Sailboat & 2.11 & 1.68 \\
\hline
\end{tabular}

228 


\begin{tabular}{|c|c|c|c|}
\hline & Boat & 2.30 & 1.20 \\
\hline & Tiffany & 4.52 & 1.24 \\
\hline Outside & Lenna & 2.94 & 1.63 \\
\hline & Family & 1.93 & 1.26 \\
\hline & Zelda & 3.47 & 1.18 \\
\hline
\end{tabular}

\section{Conclusion}

This paper has introduced a comparison among classical and well-known techniques such as VQ with different neural paradigms such as BPNN, self-organizing feature maps, which can be efficiently used for image compression purposes. Nevertheless, the full potential of the neural approaches will only be exploited completely when massively parallel implementations of such approaches are available. Moreover, in comparison to the ordinary VQ and SOFM-VQ schemes, the BPNN scheme can obtain better image quality and better visual quality about the edge region. A better PSNR performance is achieved. From the experimental results, we find that BPNN is superior to the VQ and SOFM-VQ. 


\section{REFERENCES}

[1] Abdel-Wahhab, O., and Fahmy, M. M., 1997, "Image compression using multilayer neural network," IEEE proc. V is Signal Processing, vol. 144, No. 5, October.

[2] Charif, H. Nait, and Salam, Fathi M., 2000, "Neural network-based image compression system," Proc. $43^{\text {rd }}$ IEEE Midwest symp. On circuits and systems, Lansing MI.

[3] Cottrell G, W, Munro P and Zipser D 1989 Image compression by back propagation: an example of extensional programming ICS Report 8702.

[4] Dony, Report D., and Haykin, Simon, 1995, "neural network approaches to image compression," Proc. Of the IEEE, vol. 83, No. 2, February.

[5] Gersho A, Ramstad T and Versvik I 1980 Fully vectorquantized subband coding with adaptive code book allocation Proc. IEEE Int. Conf. On Acoustics, Speech, Signal Processing (New York: IEEE Press) pp 1580-9.

[6] Linde Y, Buzo A and Gary R M 1980 An algorithm for vector quantization design IEEE Trans. Commun. C-28 84-95.

[7] Lippman Richard P., 1987. An Introduction to Computing with Neural Nets, IEEE on ASSP Magazine, Vol. 4, No. 2, April.

[8] McCollum, peter, 1997. An Introduction of Backpropagation Neural Network, from internet, Saipan 59 @juno. Com.

[9] Nasrabadi N and Feng $\mathrm{Y} 1988$ Vector quantization of image based upon a neural-network clustering algorithm SPIEVCIP 1001 207-13. 
[10] Patnaik, Suprava and Pal, R. N., 2001, "Image compression using Auto associative neural network and embedded zero-tree coding," Third IEEE processing workshop advances in wireless communication, Taiwan, March.

[11] Verma, B., 1997, Fast training of Multilayer perceptrons, IEEE Trans. On Neural Network, Vol. 8, No. 6, pp 13141321.

[12] Watanabe, Eiji, and Mori, Katsumi, 2001, "Lossy image compression using a modular structured neural Network," Proc. of IEEE signal processing society workshop, pp. 403-412. 